\title{
Fetal cardiac function by mitral and tricuspid annular plane systolic excursion using spatio-temporal image correlation M-mode and left cardiac output in fetuses of pregestational diabetic mothers
}

\author{
Nathalie Jeanne Magioli Bravo-Valenzuela, $\mathrm{PhD}^{1}$, Alberto Borges Peixoto, $\mathrm{PhD}^{2,3}$, Rosiane Mattar, $\mathrm{PhD}^{4}$, \\ Edward Araujo Júnior, $\mathrm{PhD}^{4}$

\begin{abstract}
${ }^{1}$ Pediatric Cardiology Service, Department of Pediatrics, Federal University of Rio de Janeiro (UFRJ), Rio de Janeiro-RJ; ${ }^{2}$ Gynecology and Obstetrics Service, Mario Palmério University Hospital - University of Uberaba (UNIUBE), Uberaba-MG, ${ }^{3}$ Department of Obstetrics and Gynecology, Federal University of Triângulo Mineiro (UFTM), Uberaba-MG, ${ }^{4}$ Department of Obstetrics, Paulista School of Medicine - Federal University of São Paulo (EPM-
\end{abstract} \\ UNIFESP), São Paulo-SP, Brazil
}

\section{Objective}

To assess the mitral and tricuspid annular plane systolic excursions (MAPSE and TAPSE, respectively) and cardiac output (CO) in fetuses of pregnant women with pregestational diabetes mellitus (DM) using spatio-temporal image correlation M-mode (STIC-M) and virtual organ computer-aided analysis (VOCAL).

\section{Methods}

This study was prospective and cross-sectional. It included 45 fetuses each from mothers with pregestational DM and healthy mothers, with gestation ages ranging from 20 to 36.6 weeks. The fetal cardiac volumes were obtained and analyzed by STIC and VOCAL methods. MAPSE and TAPSE were measured by STIC-M in the apical or basal fourchamber view. The values of the right (RV) and left ventricular (LV) CO were calculated by STIC and VOCAL.

\section{Results}

The median values of TAPSE were 6.1 and $6.2 \mathrm{~mm}$ in the diabetic and control groups $(P<0.001)$, respectively. The median values of MAPSE were $4.6 \mathrm{~mm}$ in the fetuses of mothers with diabetes and $4.8 \mathrm{~mm}$ in fetuses of healthy mothers. The fetal LV CO (60.4 L/min vs. $71.1 \mathrm{~L} / \mathrm{min} ; P=0.033$, respectively) and RV CO (65.2 vs. $70.1 \mathrm{~L} / \mathrm{min} ; P=0.026$, respectively) were lower in the pregestational DM group than in the control group. A significant effect of pregestational DM was observed in all functional parameters after adjusting, with fetal heart rate as covariant. There was moderate significant positive correlation between MAPSE and LV CO $(r=0.53 ; P=0.0001)$ and between TAPSE and RV CO $(r=0.46 ; P=0.0001)$.

\section{Conclusion}

Significant difference in functional parameters (TAPSE, MAPSE and LV CO) obtained by STIC and VOCAL were observed in the fetuses of the pregestational DM group compared to those of the control group.

Keywords: Fetal heart; Diabetes mellitus; Systolic heart failure; Four-dimensional echocardiography; Cardiac output

Received: 2020.09.11. Revised: 2020.12.06. Accepted: 2020.12.09.

Corresponding author: Edward Araujo Júnior, $\mathrm{PhD}$

Department of Obstetrics, Paulista School of Medicine - Federal University of São Paulo (EPM-UNIFESP), Rua Belchior de Azevedo, 156 apto. 111 Torre Vitoria, São Paulo-SP, CEP 05089-030, Brazil

E-mail: araujojred@terra.com.br, https://orcid.org/0000-0002-6145-2532

Edward Araujo Júnior has been an Editorial Board of Obstetrics \& Gynecology Science; however, he was not involved in the peer reviewer selection, evaluation, or decision process of this article. Otherwise, no other potential conflicts of interest relevant to this article was reported.

Articles published in Obstet Gynecol Sci are open-access, distributed under the terms of the Creative Commons Attribution Non-Commercial License (http://creativecommons.org/ licenses/by-nc/3.0/) which permits unrestricted non-commercial use, distribution, and reproduction in any medium, provided the original work is properly cited. 


\title{
Obstetrics \& Gynecology Science
}

\author{
Vol. 64, No. 3, 2021
}

\section{Introduction}

Classically, gestational diabetes mellitus (DM) is the main cause of cardiac hypertrophy in fetuses. Pregestational DM affects structural and functional cardiogenesis, especially when the maternal blood glucose is uncontrolled [1-4]. Studies have demonstrated that fetuses of diabetic mothers may present signs of cardiac dysfunction even in the absence of septal hypertrophy $[5,6]$. It is, therefore, important to assess the functional parameters of ultrasound/echocardiography to detect subclinical cardiac dysfunction in this population $[7,8]$.

Traditionally, the global cardiac systolic function can be assessed by shortening fraction (SF) using two-dimensional (2D) ultrasound or by ejection fraction (EF) using three- (3D) and four- (4D) dimensional ultrasound with spatio-temporal image correlation (STIC) and virtual organ computer-aided analysis (VOCAL) methods $[9,10]$. SF is applied to evaluate the global radial contractility by measuring the reduction of the ventricular diameter of end-diastole to end-systole $[11,12]$. Using advanced techniques such as 3D ultrasound, the EF reflects the percentage of blood ejected by the ventricles in each cardiac cycle and can be used to evaluate the longitudinal myocardial function with good reproducibility $[10,13,14]$.

The longitudinal cardiac contractility can be assessed by measuring the maximum mitral and tricuspid annular plane systolic excursions (MAPSE and TAPSE, respectively). These parameters can be affected in the early stages of cardiac dysfunction and can be obtained by means of ultrasound using conventional anatomical M-mode tracings and by STIC Mmode (STIC-M) [15]. Studies on fetal TAPSE and MAPSE have demonstrated the applicability of these parameters on risk factors for fetal heart failure, such as fetal growth restriction and maternal DM [16-18].

The aim of this study was to compare the values of fetal MAPSE, TAPSE, and cardiac output (CO) obtained by STIC-M and VOCAL in fetuses from pregnant women with DM and controls.

\section{Materials and methods}

This study was prospective and cross-sectional, performed to evaluate fetal MAPSE, TAPSE, and CO in fetuses from diabetic mothers compared with those in fetuses from lowrisk pregnant women between 20 and 36.6 weeks of ges- tation. From November 2016 to November 2018, all data underwent routine ultrasound evaluation and fetal echocardiogram at the same time at the Fetal Cardiology Sector of the Federal University of São Paulo (UNIFESP).

The DM and control groups included fetuses of pregnant women with pregestational DM types 1 , 2, or maturityonset diabetes of the young and those of healthy mothers, respectively. The inclusion criteria were as follows: singleton pregnancies with gestational age (GA) between 20 and 36.6 weeks determined by the last menstrual period and confirmed by first trimester ultrasound. The exclusion criteria were as follows: fetuses whose mothers had other comorbidities except pregestational DM; estimated fetal weight $<10$ th percentile for GA; smokers or drug/ alcohol users; fetuses with structural cardiac and extracardiac anomalies at ultrasound examination; and fetal cardiac volumes with inadequate quality images due to fetal movements, presence of acoustic shadows of fetal ribs or spine, and maternal breathing.

Pregnant women were assessed once. The obstetrical ultrasound and fetal echocardiogram examinations were performed on the same day using a Voluson E6 apparatus (General Electric Healthcare, Zipf, Austria) equipped with 3.0-5.0 MHz convex volumetric probe. Examinations were performed by two examiners (NJMBV and ABP) with experience in both 3D ultrasound and fetal echocardiography. First, evaluation of the fetal morphology and biometry were assessed by 2D ultrasound. Fetal cardiac volumes were acquired in the four-chamber view according to Gonçalves et al. [19]. The angle of the scan acquisition was standardized, ranging from $20^{\circ}$ to $25^{\circ}$ and from $35^{\circ}$ to $40^{\circ}$ in the second and third trimesters, respectively, with a speed time ranging from 7.5 to 15 seconds. One to three fetal cardiac volumes were obtained from each pregnant woman and stored in DICOM. The off-line analysis was performed by the principal investigator (NJMBV) using 4D view software version 10.0 (General Electric Medical System, Zipf, Austria). The axial plane $(A)$ of the multiplanar mode was selected, and the fetal heart was rotated around the ' $z$ ' axis for the fetal spine to be at a 6 o'clock position. Image adjustments with sepia or candle colors and cineloop mode were reduced to $6 \%$. To measure fetal MAPSE and TAPSE, the STIC-M key was activated and the cursor was placed at the atrioventricular junction, marked by mitral and tricuspid valves, respectively. The maximum amplitude of motion was obtained as per the extent of displacement between end-systole and end- 


\section{Obstetrics \& Gynecology Science}

Nathalie Jeanne Magioli Bravo-Valenzuela, et al. Fetal MAPSE, TAPSE, and LV CO

diastole (measured in $\mathrm{mm}$ ) (Fig. 1). The maximal and minimal ventricular volumes were measured at the end-diastole (just after closing of the atrioventricular valves) and end-systole (before opening of the atrioventricular valves), respectively.

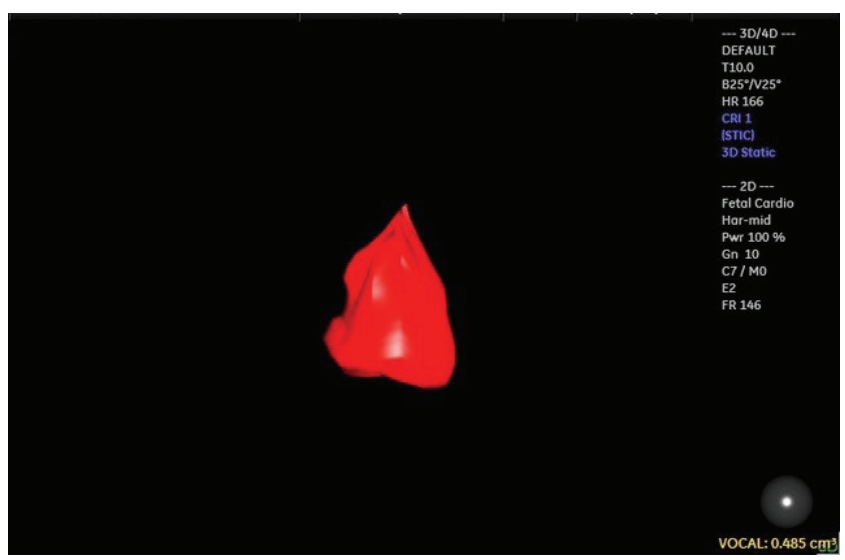

Fig. 1. Measurement of the fetal left ventricle (LV) volume using spatio-temporal image correlation and virtual organ computeraided analysis (VOCAL) methods in a fetus from pregestational diabetes mellitus at 22 weeks and 5 days of gestation (LV diastolic volume $=0.48 \mathrm{~cm}^{3}$ and heart rate $\left.[\mathrm{HR}]=166 \mathrm{bpm}\right)$. The LV cardiac output (LV CO) and LV ejection fraction (LVEF) were calculated from the measurements of the ventricular volumes according to the following formulas: LV CO=(LVDV-LVSV) $\times H R$ and LVEF (LVDVLVSV)/LVDV. The calipers were positioned on the internal border of the mitral valve and on the ventricular endocardial border to perform the manual delimitation of the internal left ventricular volume. After 6 sequential planes, the VOCAL software provided the chamber volume and the reconstructed 3D image. LVDv, left diastolic volume; LVSv, left systolic volume.
Subsequently, the caliper was positioned in the atrioventricular valve and in the ventricular apical region (Fig. 2). Then, the VOCAL key was activated, and this software provided the reconstruction of the 3D image of each ventricle after 6 sequential manual planes $\left(30^{\circ}\right)$. The EF and $\mathrm{CO}$ for left and right ventricles were calculated using the following formulas: $\mathrm{EF}=$ end diastolic ventricular volume-end systolic ventricular volume/end diastolic ventricular volume and $\mathrm{CO}=$ end diastolic ventricular volume-end systolic ventricular volumexheart rate.

The following clinical variables were also collected to analyze the characteristics of the study population: maternal age, number of previous pregnancies, parity, number of miscarriages, GA at the time of ultrasound examination, estimated fetal weight, fetal heart rate (FHR), and last serum glycated hemoglobin levels.

Data were transferred to an Excel 2010 spreadsheet (Microsoft Corp., Redmond, WA, USA) and analyzed using PASW version 20.0 (SPSS Inc., Chicago, IL, USA) and GraphPad (version 7.0, GraphPad Software, San Diego, CA, USA). Continuous variables were expressed as the medians (minimum and maximum ranges). Categorical variables were expressed as percentages. The Mann-Whitney $U$-test was used to compare the two groups, with a critical alpha of 0.05 . Spearman's correlation test ( $r$ ) was used to determine the correlation between continuous variables. Linear regression was used to analyze the influence of the FHR on fetal cardiac function parameters, the influence of MAPSE on the left ventricle (LV) $\mathrm{CO}$, and the influence of TAPSE on the right ventricle (RV)
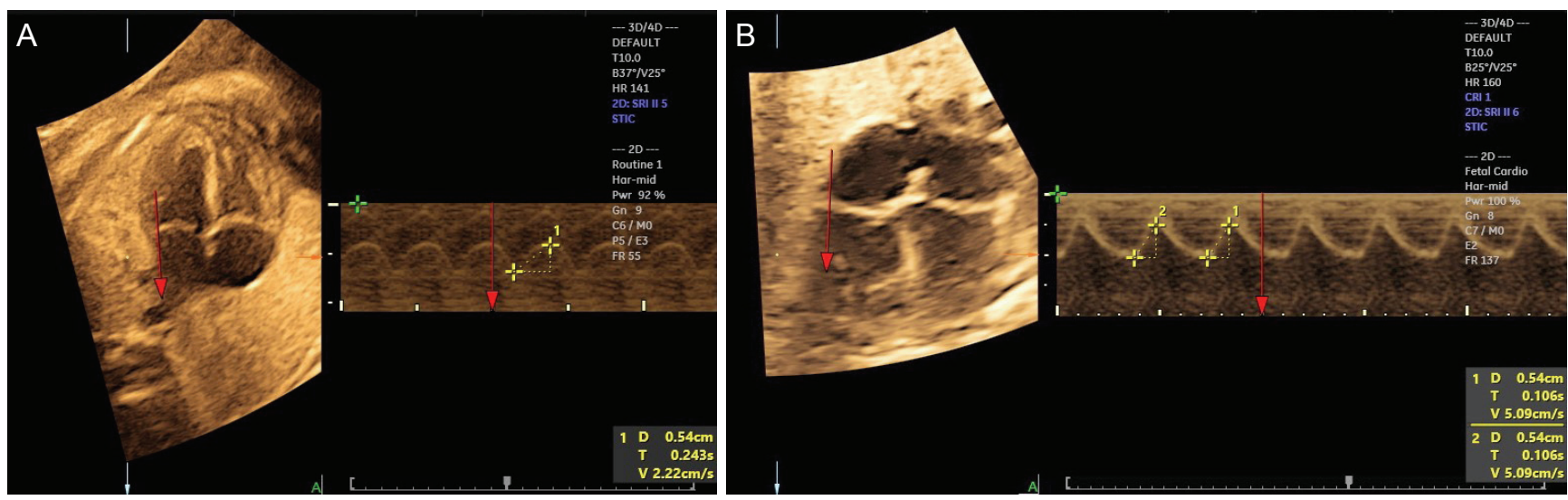

Fig. 2. In the apical four-chamber view of the fetal heart, the spatio-temporal image correlation in M-mode image is positioned at the junction between the free ventricular wall and the atrioventricular valve for the calculation of mitral annular plane systolic excursion (MAPSE) (A) and tricuspid annular plane systolic excursion (TAPSE) (B) annular plane systolic excursions. MAPSE=5.4 mm; TAPSE=5.4 mm. 


\section{Obstetrics \& Gynecology Science}

Vol. 64, No. 3, 2021

CO. General linear model with FHR as covariant was used to evaluate the influence of pregestational DM on fetal cardiac parameters.

\section{Results}

We evaluated 104 pregnant women. However, 13 fetuses were excluded because of the poor quality of the fetal cardiac volume acquisition, 1 case because the estimated fetal weight was $<10$ th percentile for $G A$, and 2 cases because of fetal structural anomalies. Consequently, we evaluated a total of 90 fetal cardiac volumes, which were divided in two groups (45 fetuses each from mothers with pregestational DM and healthy mothers).

There was a significant effect of pregestational diabetes on maternal age $(P=0.004)$, number of previous pregnancies $(P<0.01)$, parity $(P=0.005)$, and number of miscarriages $(P=0.001)$. Healthy pregnant women had higher maternal age (33.0 vs. 27.0 years, $P=0.004$ ) and lower number of previous pregnancies (1.0 vs. 2.0, $P<0.001$ ) than pregnant women with pregestational DM (Table 1).

Considering all cases included in the study, there was a weak significant negative correlation between FHR and the following: TAPSE ( $r=-0.37, P=0.0003)$, MAPSE $(r=-0.31$, $P=0.0029), \operatorname{LV} C O(r=-0.29, P=0.0049)$, and RV CO $(r=-0.30$, $P=0.0035)$ (Table 2). The elevation of 1 beat per minute (bpm) in the FHR was responsible for reducing TAPSE by $0.04 \mathrm{~mm}$, MAPSE $0.03 \mathrm{~mm}$, LV CO $0.79 \mathrm{~L} / \mathrm{min}$, and RV CO $0.94 \mathrm{~L} / \mathrm{min}$. According to the coefficient of determination $\left(R^{2}\right), 15 \%$, $12 \%, 5 \%$, and $6 \%$ of the variations in TAPSE, MAPSE, LV $\mathrm{CO}$, and $\mathrm{RV} C \mathrm{C}$, respectively, were linearly related to FHR, with the remaining variations resulting from other factors not considered in the model (Table 2 and Fig. 3). Considering all cases of mothers with pregestational DM, whose glycated hemoglobin (HB) (\%) measurements had been performed, there was a weak significant negative correlation with TAPSE $(r=-0.37, P=0.019)$ and MAPSE $(r=-0.38, P=0.015)$. There was not significant correlation between glycated $\mathrm{HB}(\%)$ and LV CO ( $r=0.04, P=0.783)$ and RV CO ( $r=0.03, P=0.819)$ (Table 3).

Table 1. Clinical characteristics of the studied population

\begin{tabular}{|c|c|c|c|c|c|c|c|}
\hline \multirow{2}{*}{ Characteristics } & \multicolumn{4}{|c|}{ Control $(n=45)$} & \multicolumn{3}{|c|}{ Pregestational diabetes mellitus $(n=45)$} \\
\hline & Median & Min & Max & Median & Min & Max & $P$-value ${ }^{\text {a) }}$ \\
\hline Age (yr) & 33.0 & 19.0 & 43.0 & 27.0 & 17.0 & 43.0 & 0.004 \\
\hline Previous pregnancy number & 1.0 & 1.0 & 2.0 & 2.0 & 1.0 & 6.0 & $<0.001$ \\
\hline Parity & 0.0 & 0.0 & 3.0 & 0.0 & 0.0 & 3.0 & 0.005 \\
\hline Previous miscarriage number & 0.0 & 0.0 & 1.0 & 0.0 & 0.0 & 5.0 & 0.001 \\
\hline $\mathrm{GA}(w k)$ & 27.3 & 20.0 & 33.3 & 27.5 & 20.2 & 34.6 & 0.831 \\
\hline EFW (g) & 978.0 & 322.0 & $2,137.0$ & $1,152.0$ & 333.0 & $2,689.0$ & 0.407 \\
\hline FHR (bpm) & 144.0 & 121.0 & 160.0 & 144.0 & 113.0 & 166.0 & 0.577 \\
\hline Glycate HB (\%) & & & & & 7.6 & 4.8 & 15.5 \\
\hline
\end{tabular}

Min, minimum; Max, maximum; GA, gestational age; EFW, estimated fetal weight; FHR, fetal heart rate; HB, hemoglobin.

a) Mann-Whitney, $P<0.05$.

Table 2. Influence of fetal heart rate (bpm) on parameters for assessing fetal cardiac function

\begin{tabular}{lccccc}
\hline Variables & Equation & $\mathbf{r}$ & $\mathbf{R}^{2}$ & $\boldsymbol{P}_{\text {-value }}^{\text {a) }}$ & $\boldsymbol{P}_{\text {-value }}$ \\
\hline TAPSE $(\mathrm{mm})$ & $\mathrm{Y}=-0.0418^{\text {b) }} \mathrm{X}+12.21$ & -0.38 & 0.15 & 0.0003 & 0.0002 \\
MAPSE volume $(\mathrm{mm})$ & $\mathrm{Y}=-0.03121 * X+9.395$ & -0.31 & 0.12 & 0.0029 & 0.0008 \\
LV cardiac output $(\mathrm{L}$ min) & $\mathrm{Y}=-0.7961 * X+184.6$ & -0.29 & 0.05 & 0.0049 & 0.0304 \\
RV cardiac output (L/min) & $Y=-0.9448 * X+209.2$ & -0.30 & 0.06 & 0.0035 & 0.0233 \\
\hline
\end{tabular}

TAPSE, tricuspid annular plane systolic excursion; MAPSE, mitral annular plane systolic excursion; LV, left ventricle; RV, right ventricle, $Y$, fetal cardiac function parameter; $X$, fetal heart rate (bpm); $r$, Spearman's correlation coefficient; $R^{2}$, coefficient of determination.

a) Spearman correlation test; ${ }^{b}$ Linear regression, $P<0.05$. 


\section{Obstetrics \& Gynecology Science}

Nathalie Jeanne Magioli Bravo-Valenzuela, et al. Fetal MAPSE, TAPSE, and LV CO

There was a significant effect of pregestational DM on TAPSE $(P<0.001)$, MAPSE $(P<0.001)$, LV CO $(P=0.033)$, and RV CO $(P=0.026)$ after adjusting, with FHR as covariant. The median TAPSE (6.1 vs. $6.2 \mathrm{~mm}, P<0.001$, respectively),
MAPSE (4.6 vs. $4.8 \mathrm{~mm}, P<0.001$, respectively), LV CO (60.4 vs. $71.1 \mathrm{~L} / \mathrm{min}, P=0.033$, respectively), and RV CO (65.2 vs. $70.1 \mathrm{~L} / \mathrm{min}, P=0.026$, respectively) were lower in the pregestational DM group than in the control group (Table 4).
A

$$
Y=-0.0418 * X+12.21, r=-0,38, R^{2}=0,148, P=0,0002
$$

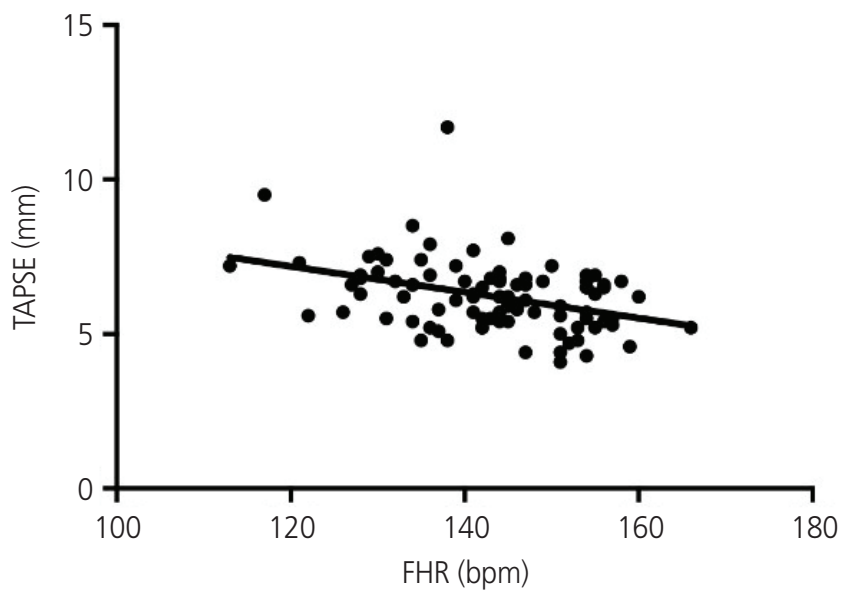

C

$$
Y=-0.7961 * X+184.6, r=-0,29, R^{2}=0,052, P=0,030
$$

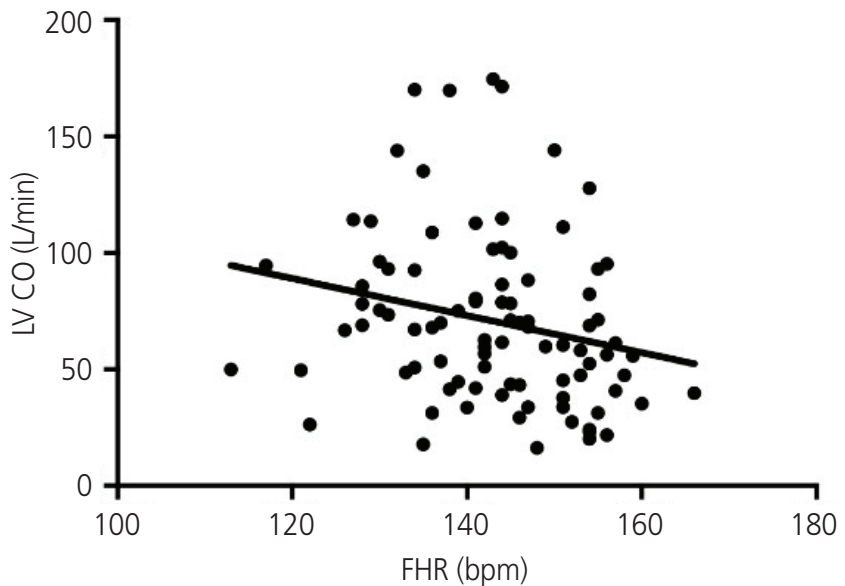

B $Y=-0.03121 * X+9.395, r=-0,31, R^{2}=0,119, P=0,0008$

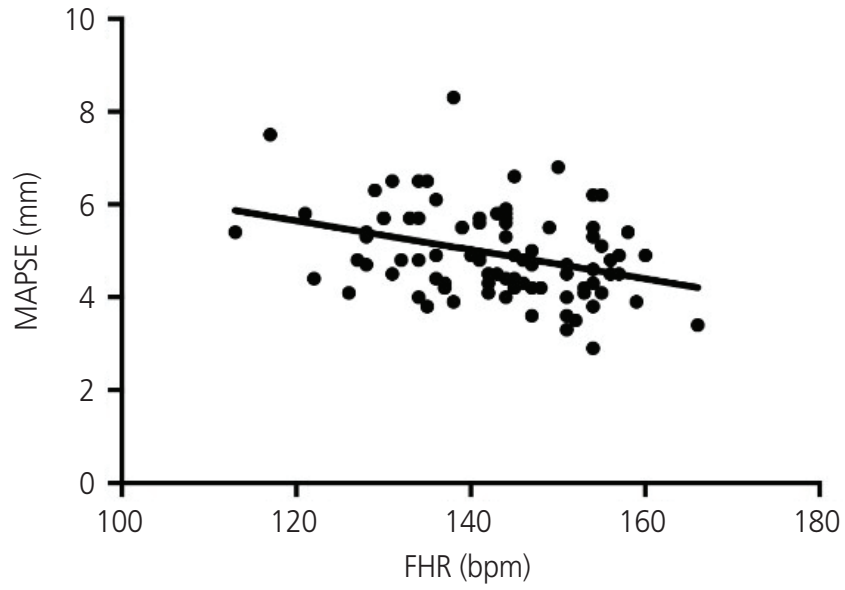

D

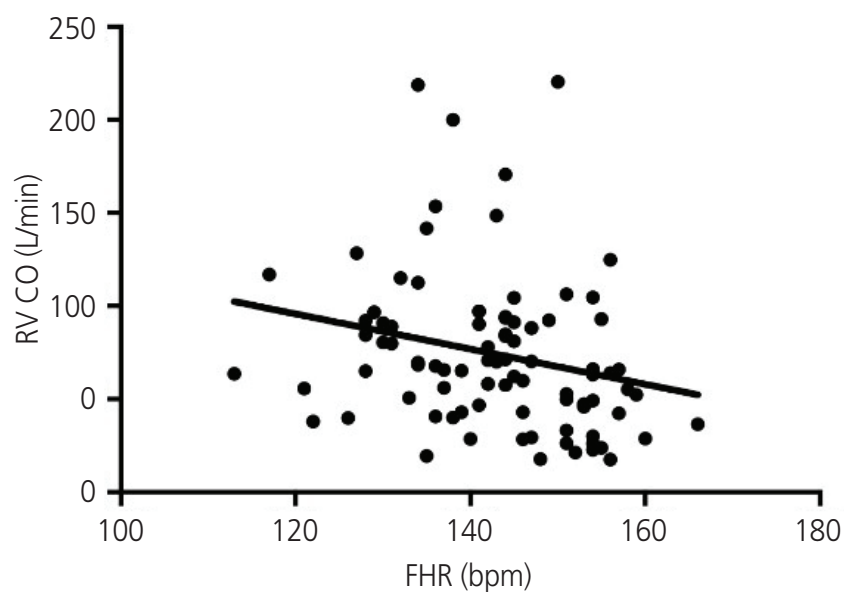

Fig. 3. Scatter plot of the correlation between tricuspid annular plane systolic excursion (TAPSE) (A), mitral annular plane systolic excursion (MAPSE) (B), left ventricle cardiac output (LV CO) (C), right ventricle cardiac output (RV CO) (D), and fetal heart rate (FHR).

Table 3. Influence of glycated hemoglobin on parameters for assessing fetal cardiac function

\begin{tabular}{lccccc}
\hline Variables & Equation & $\mathbf{r}$ & $\mathbf{R}^{\mathbf{2}}$ & $\boldsymbol{P}$-value & $\boldsymbol{P}^{\text {a) }}$ \\
\hline TAPSE $(\mathrm{mm})$ & $\mathrm{Y}=-0.09602 * X+7.123$ & -0.37 & 0.053 & 0.019 & 0.250 \\
MAPSE volume $(\mathrm{mm})$ & $\mathrm{Y}=-0.081{ }^{*}{ }^{\mathbf{b})} \mathrm{X}+5.596$ & -0.38 & 0.043 & 0.015 & 0.204 \\
LV cardiac output $(\mathrm{L} / \mathrm{min})$ & $\mathrm{Y}=1.402 * X+74.97$ & 0.04 & 0.001 & 0.783 & 0.836 \\
RV cardiac output $(L / \mathrm{min})$ & $\mathrm{Y}=0.4514 * X+69.56$ & 0.03 & 0.000 & 0.819 & 0.863 \\
\hline
\end{tabular}

TAPSE, tricuspid annular plane systolic excursion; MAPSE, mitral annular plane systolic excursion; LV, left ventricle; RV, right ventricle, $Y$, fetal cardiac function parameter; $X$, fetal heart rate (bpm); $r$, Spearman's correlation coefficient; $R^{2}$, coefficient of determination.

${ }^{\text {a) }}$ Spearman correlation test; ${ }^{b}$ Linear regression, $P<0.05$. 


\section{Obstetrics \& Gynecology Science}

Vol. 64, No. 3, 2021

Considering all included cases, there was moderate significant positive correlation between MAPSE and LV CO $(r=0.53$, $P<0.0001)$ and TAPSE $(r=0.46, P<0.0001)$ and RV CO (Table 5). The 1-mm increase in the excursion of LV annulus was responsible for increasing the LV CO by $21.56 \mathrm{~L} / \mathrm{min}$ and RV CO by $19.62 \mathrm{~L} / \mathrm{min}$. According to the $\mathrm{R}^{2}, 31 \%$ and $28 \%$ of the variations in LV CO and RV CO were linearly related to MAPSE and TAPSE, respectively, with the remaining variations resulting from other factors not considered in the model (Table 5 and Fig. 4).

\section{Discussion}

TAPSE and MAPSE measurements can be obtained either by 2D ultrasound using anatomical M-mode tracings or by ad-

Table 4. Fetal cardiac functional parameters by spatio-temporal image correlation in M-mode and virtual organ computer-aided analysis

\begin{tabular}{|c|c|c|c|c|c|c|c|c|c|c|}
\hline \multirow{2}{*}{ Variables } & \multicolumn{3}{|c|}{ Control $(n=45)$} & \multicolumn{7}{|c|}{ Pregestational diabetes mellitus $(n=45)$} \\
\hline & Median & Min & Max & Median & Min & Max & $F$ & $n^{2}$ & $P$-value ${ }^{\text {a) }}$ & $P$-value ${ }^{b)}$ \\
\hline TAPSE $(\mathrm{mm})$ & 6.2 & 4.1 & 8.5 & 6.1 & 4.3 & 11.7 & 7.7 & 0.2 & 0.640 & $<0.001$ \\
\hline MAPSE (mm) & 4.8 & 3.6 & 6.8 & 4.6 & 2.9 & 8.3 & 6.2 & 0.1 & 0.260 & $<0.001$ \\
\hline LV CO (L/min) & 71.1 & 16.3 & 170.1 & 60.4 & 17.7 & 174.5 & 2.8 & 0.1 & 0.111 & 0.033 \\
\hline RV CO (L/min) & 70.1 & 17.8 & 220.5 & 65.2 & 17.6 & 200.1 & 3.2 & 0.1 & 0.247 & 0.026 \\
\hline
\end{tabular}

Min, minimum; Max, maximum; F, F ratio; $\mathrm{n}^{2}$, Eta square; TAPSE, tricuspid annular plane systolic excursion; MAPSE, mitral annular plane systolic excursion; LV CO, left ventricle cardiac output; RV CO, right ventricle cardiac output.

${ }^{\text {a) }}$ Mann-Whitney test; ${ }^{\text {b) }}$ General linear model, $P<0.05$.

Table 5. Influence of tricuspid annular plane systolic excursion on RV cardiac output and influence of mitral annular plane systolic excursion on LV cardiac output

\begin{tabular}{|c|c|c|c|c|c|}
\hline Variables & Equation & $r$ & $\mathbf{R}^{2}$ & $P$-value ${ }^{\text {a) }}$ & $P$-value ${ }^{\text {b) }}$ \\
\hline LV cardiac output (L/min) & $Y=21.56 * X-35.63$ & 0.53 & 0.31 & $<0.0001$ & $<0.0001$ \\
\hline RV cardiac output (L/min) & $Y=19.62 * X-48.06$ & 0.46 & 0.28 & $<0.0001$ & $<0.0001$ \\
\hline
\end{tabular}

$R V$, right ventricle; LV, left ventricle; r, Spearman's correlation coefficient; $R^{2}$, coefficient of determination; $Y$, LV or RV cardiac output (L/min); $X$, MAPSE or TAPSE (mm); MAPSE, mitral annular plane systolic excursion; TAPSE, tricuspid annular plane systolic excursion.

a)Spearman correlation test; b) Linear regression.

$$
\text { A }
$$

$$
Y=21.56 * X-35.63, r=0,53, R^{2}=0,31, P<0.0001
$$

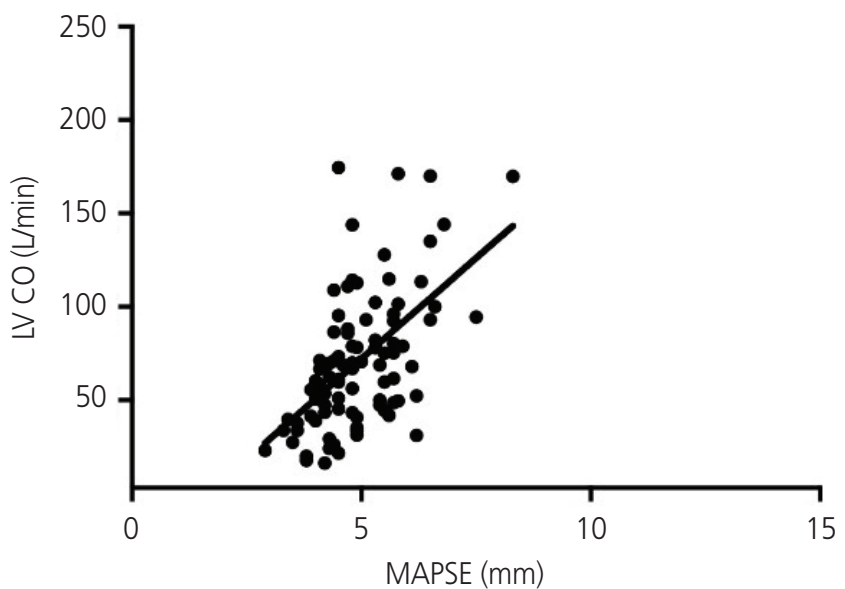

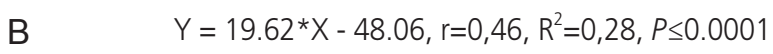

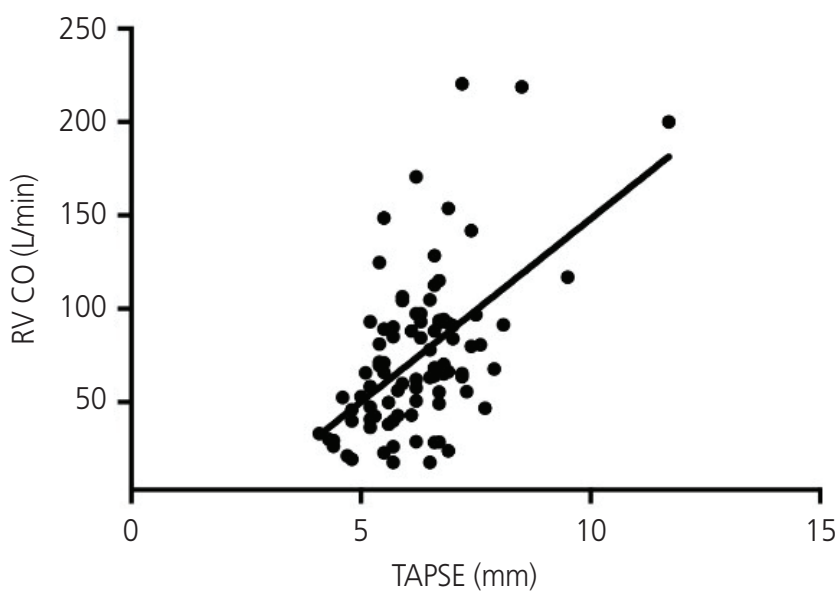

Fig. 4. Scatter plot of the correlation between mitral annular plane systolic excursion (MAPSE) and left ventricle cardiac output (LV CO) (A) and correlation between tricuspid annular plane systolic excursion (TAPSE) and right ventricle cardiac output (RV CO) (B). 


\section{Obstetrics \& Gynecology Science}

Nathalie Jeanne Magioli Bravo-Valenzuela, et al. Fetal MAPSE, TAPSE, and LV CO

vanced methods such as 3D/4D ultrasound with STIC (STIC-M) [20]. Traditionally, it was demonstrated that the conventional 2D M-mode is a simple and useful technique for the assessment of the longitudinal systolic cardiac function in fetuses. Fetal reference ranges for this parameter were established [20-23]. Nevertheless, some authors have demonstrated the advantages of the STIC-M over the conventional M-mode. The 3D/4D ultrasound STIC enables the optimization of the post-processed images by rotating the fetal cardiac volumes, minimizing limitations addressed to an unfavorable fetal position, and increasing the accuracy of the TAPSE and MAPSE measurements $[17,24]$. Moreover, other functional parameters such as $C O$ and EF, which can be obtained with good reproducibility using 3D/4D ultrasound methods and reference curves, have been validated [13,25-27].

Messing et al. [17] demonstrated that it was possible to obtain the measurements of fetal TAPSE by 3D ultrasound with STIC, and their measurement values were similar to those obtained by the anatomical 2D M-mode. These authors included a large sample size and provided the first reference ranges of fetal TAPSE by STIC with 341 low-risk singleton pregnant women. They demonstrated that the STIC Mmode fetal TAPSE increased linearly with the GA, with mean values ranging from $4.2 \mathrm{~mm}$ at 21 to $8.8 \mathrm{~mm}$ at 39 weeks of gestation. In our study, we observed a positive correlation between fetal TAPSE and the GA, with a mean of $4.1 \mathrm{~mm}$ at 20 and $8.5 \mathrm{~mm}$ at 36 weeks of gestation in healthy fetuses.

Tedesco et al. [18] determined reference ranges for fetal TAPSE using STIC-M in 300 fetuses between 20 and 39 weeks of gestation and observed linear increase with GA. These authors observed that the fetal TAPSE values were lower in fetuses of mothers with pregestational DM than in fetuses of healthy mothers. Furthermore, in Tedesco's study, the parameter used to assess the global systolic cardiac function was the SF obtained by STIC-M. Significant differences were observed between the two groups. Similarly, in this study, the median values of fetal TAPSE by STIC-M were lower in fetuses of mothers with pregestational DM than in fetuses of healthy pregnant women. In our study, the global systolic cardiac evaluation was assessed by CO using STIC and VOCAL. A significant effect of pregestational DM on the measurements of RV and LV CO was observed after adjusting, with FHR as a covariant.

Interestingly, in other cross-sectional studies from our group using STIC and VOCAL, no significant differences in
$\mathrm{CO}$ and EF were observed between the fetuses of pregnant women with pregestational DM and the fetuses of healthy mothers. However, significant differences in fetal left atrial volumes between the two groups were observed. This could be related to left atrial dysfunction, reflecting an earlier stage of cardiac dysfunction (left heart diastolic dysfunction) [28]. Alternatively, in this study, the LV and RV CO were lower in fetuses of those in the pregestational DM group than in the fetuses of those in the control group. We believe that this difference could be related to the inclusion of pregnant women with gestation periods of up to 36 weeks.

Since 2004, various studies showed that the CO calculated using 3D/4D ultrasound was positively correlated to the GA $[13,27,29]$. Accordingly, we observed an increase in the RV $\mathrm{CO}$ and LV CO values with the GA. Furthermore, the parameters that we used to assess the longitudinal and radial cardiac functions were correlated positively (TAPSE and MAPSE with RV CO and LV CO, respectively).

MAPSE reflects systolic longitudinal LV function as a useful tool in the assessment of the LV function in a variety of situations that may evolve with risks for fetal heart failure, such as maternal DM. However, limited studies on the reference values of fetal MAPSE in low-risk population have been published until now $[20,23]$. Recently, reference values were established for fetal MAPSE, TAPSE, and septum annular plane systolic excursion (SAPSE) using 2D and M-mode between 20 and 36.6 weeks of gestation in low-risk pregnant women. The mean of each parameter ranged as follows: SAPSE, 2.87-5.56 mm; MAPSE, 3.98-8.07 mm; and TAPSE, $2.34-4.21 \mathrm{~mm}$. There was a significant positive correlation between these parameters and GA [30].

To the best of our knowledge, this was the first study that assessed MAPSE using STIC-M in fetuses of mothers with pregestational DM. Atiq et al. [31] performed a study of fetal MAPSE using the conventional 2D M-mode in mothers with gestational diabetes (64 cases and 64 controls). The values of MAPSE were lower in the diabetic group than in the normal group (4.8 mm in DM vs. $5.0 \mathrm{~mm}$ in controls). Our results of fetal MAPSE using STIC-M were similar to those of Atiq et al. [31], with mean MAPSE values of 4.8 in the pregestational DM group and $5.0 \mathrm{~mm}$ in the control group. The differences in fetal MAPSE between the two groups reflect the effect of DM on LV function. In this study, significant differences between fetal MAPSE, TAPSE, and CO were observed in mothers with pregestational DM and healthy mothers, reflecting 


\section{Obstetrics \& Gynecology Science}

Vol. 64, No. 3, 2021

changes in the cardiac function.

The limitations of this study were the small number of cases. Additionally, the comparison between DM and control groups based on GA using Z-score or standard deviation were not done. In addition, we did not compare the values according to each trimester of pregnancy as well as between well and poorly controlled DM. Furthermore, the postnatal outcomes were not available.

In conclusion, fetal MAPSE, TAPSE, and CO using STIC-M and VOCAL could be applied and may assist fetal echocardiographers in the antenatal surveillance of fetuses with high-risk conditions for fetal heart failure, such as mothers with pregestational DM.

\section{Conflict of interest}

No potential conflict of interest relevant to this article was reported.

\section{Ethical approval}

The study was approved by the Ethic Committee of UNIFESP (CAAE: 87111116.4.0000.5505).

\section{Patient consent}

All participants provided a consent form.

\section{Funding information}

None.

\section{References}

1. Turan S, Turan OM, Miller J, Harman C, Reece EA, Baschat AA. Decreased fetal cardiac performance in the first trimester correlates with hyperglycemia in pregestational maternal diabetes. Ultrasound Obstet Gynecol 2011;38:325-31.

2. Passarella G, Trifirò G, Gasparetto M, Moreolo GS, Mila- nesi O. Disorders in glucidic metabolism and congenital heart diseases: detection and prevention. Pediatr Cardiol 2013;34:931-7.

3. Rizzo G, Arduini D, Capponi A, Romanini C. Cardiac and venous blood flow in fetuses of insulin-dependent diabetic mothers: evidence of abnormal hemodynamics in early gestation. Am J Obstet Gynecol 1995;173:1775-81.

4. Han SS, Wang G, Jin Y, Ma ZL, Jia WJ, Wu X, et al. Investigating the mechanism of hyperglycemia-induced fetal cardiac hypertrophy. PLoS One 2015;10:e0139141.

5. Miranda JO, Cerqueira RJ, Ramalho C, Areias JC, Henriques-Coelho T. Fetal cardiac function in maternal diabetes: a onventional and speckle-tracking echocardiographic study. J Am Soc Echocardiogr 2018;31:33341.

6. Balli S, Pac FA, Ece I, Oflaz MB, Kibar AE, Kandemir Ö. Assessment of cardiac functions in fetuses of gestational diabetic mothers. Pediatr Cardiol 2014;35:30-7.

7. Patey O, Carvalho JS, Thilaganathan B. Perinatal changes in fetal cardiac geometry and function in diabetic pregnancy at term. Ultrasound Obstet Gynecol 2019;54:63442.

8. Mohsin M, Sadqani S, Younus K, Hoodbhoy Z, Ashiqali $S$, Atiq M. Evaluation of cardiac function in fetuses of mothers with gestational diabetes. Cardiol Young 2019;29:1264-7.

9. Rocha LA, Rolo LC, Araujo Júnior E. How to perform a functional assessment of the fetal heart: a pictorial review. Ultrasonography 2019;38:365-73.

10. Bravo-Valenzuela NJ, Peixoto $A B$, Carrilho MC, Siqueira Pontes AL, Chagas CC, Simioni $C$, et al. Fetal cardiac function by three-dimensional ultrasound using 4D-STIC and VOCAL - an update. J Ultrason 2019;19:287-94.

11. Huhta JC. Fetal congestive heart failure. Semin Fetal Neonatal Med 2005;10:542-52.

12. Tongsong T, Wanapirak C, Piyamongkol W, Sirichotiyakul S, Tongprasert F, Srisupundit K, et al. Fetal ventricular shortening fraction in hydrops fetalis. Obstet Gynecol 2011;117:84-91.

13. Molina FS, Faro C, Sotiriadis A, Dagklis T, Nicolaides KH. Heart stroke volume and cardiac output by four-dimensional ultrasound in normal fetuses. Ultrasound Obstet Gynecol 2008;32:181-7.

14. Simioni C, Nardozza LM, Araujo Júnior E, Rolo LC, Terasaka OA, Zamith MM, et al. Fetal cardiac function 


\section{Obstetrics \& Gynecology Science}

Nathalie Jeanne Magioli Bravo-Valenzuela, et al. Fetal MAPSE, TAPSE, and LV CO

assessed by spatio-temporal image correlation. Arch Gynecol Obstet 2011;284:253-60.

15. Bravo-Valenzuela NJ, Peixoto AB, Nardozza LM, Souza AS, Araujo Júnior E. Applicability and technical aspects of two-dimensional ultrasonography for assessment of fetal heart function. Med Ultrason 2017;19:94-101.

16. Cruz-Lemini $M$, Crispi F, Valenzuela-Alcaraz B, Figueras $F$, Sitges M, Gómez $\mathrm{O}$, et al. Value of annular M-mode displacement vs tissue Doppler velocities to assess cardiac function in intrauterine growth restriction. Ultrasound Obstet Gynecol 2013;42:175-81.

17. Messing B, Gilboa Y, Lipschuetz M, Valsky DV, Cohen SM, Yagel S. Fetal tricuspid annular plane systolic excursion (f-TAPSE): evaluation of fetal right heart systolic function with conventional M-mode ultrasound and spatiotemporal image correlation (STIC) M-mode. Ultrasound Obstet Gynecol 2013;42:182-8.

18. Tedesco GD, de Souza Bezerra M, Barros FS, Martins WP, Nardozza LM, Mattar R, et al. Fetal heart function by tricuspid annular plane systolic excursion and ventricular shortening fraction using STIC M-mode: reference ranges and validation. Am J Perinatol 2017;34:1354-61.

19. Gonçalves LF, Lee W, Espinoza J, Romero R. Examination of the fetal heart by four-dimensional (4D) ultrasound with spatio-temporal image correlation (STIC). Ultrasound Obstet Gynecol 2006;27:336-48.

20. Mao YK, Zhao BW, Wang B. Z-score reference ranges for angular M-mode displacement at 22-40 weeks' gestation. Fetal Diagn Ther 2017;41:115-26.

21. Carvalho JS, O'Sullivan C, Shinebourne EA, Henein MY. Right and left ventricular long-axis function in the fetus using angular M-mode. Ultrasound Obstet Gynecol 2001;18:619-22.

22. Germanakis I, Pepes S, Sifakis S, Gardiner H. Fetal longitudinal myocardial function assessment by anatomic Mmode. Fetal Diagn Ther 2012;32:65-71.

23. Lee-Tannock A, Hay K, Gooi A, Kumar S. Longitudinal reference ranges for tricuspid annular plane systolic excursion and mitral annular plane systolic excursion in normally grown fetuses. J Ultrasound Med 2020;39:929-37.

24. Godfrey ME, Messing B, Valsky DV, Cohen SM, Yagel S. Fetal cardiac function: M-mode and 4D spatiotemporal image correlation. Fetal Diagn Ther 2012;32:17-21.

25. Schoonderwaldt EM, Groenenberg IA, Hop WC, Wladimiroff JW, Steegers EA. Reproducibility of echocardiographic measurements of human fetal left ventricular volumes and ejection fractions using four-dimensional ultrasound with the spatio-temporal image correlation modality. Eur J Obstet Gynecol Reprod Biol 2012;160:22-9.

26. DeKoninck P, Steenhaut $P$, Van Mieghem T, Mhallem M, Richter J, Bernard P, et al. Comparison of Doppler-based and three-dimensional methods for fetal cardiac output measurement. Fetal Diagn Ther 2012;32:72-8.

27. Simioni C, Nardozza LM, Araujo Júnior E, Rolo LC, Zamith $\mathrm{M}$, Caetano AC, et al. Heart stroke volume, cardiac output, and ejection fraction in 265 normal fetus in the second half of gestation assessed by $4 \mathrm{D}$ ultrasound using spatio-temporal image correlation. J Matern Fetal Neonatal Med 2011;24:1159-67.

28. Bravo-Valenzuela NJ, Peixoto AB, Mattar R, Melo Júnior JF, da Silva Pares DB, Araujo Júnior E. Fetal cardiac function and ventricular volumes determined by threedimensional ultrasound using STIC and VOCAL methods in fetuses from pre-gestational diabetic women. Pediatr Cardiol 2020;41:1125-34.

29. Hamill N, Yeo L, Romero R, Hassan SS, Myers SA, Mittal $P$, et al. Fetal cardiac ventricular volume, cardiac output, and ejection fraction determined with 4-dimensional ultrasound using spatiotemporal image correlation and virtual organ computer-aided analysis. Am J Obstet Gynecol 2011;205:76.e1-10.

30. Peixoto AB, Bravo-Valenzuela NJ, Martins WP, Tonni $G$, Mattar R, Moron AF, et al. Reference ranges for the fetal mitral, tricuspid, and interventricular septum annular plane systolic excursions (mitral annular plane systolic excursion, tricuspid annular plane systolic excursion, and septum annular plane systolic excursion) between 20 and $36+6$ weeks of gestation. J Perinat Med 2020;48:601-8.

31. Atiq M, Ikram A, Hussain BM, Saleem B. Assessment of cardiac function in fetuses of gestational diabetic mothers during the second trimester. Pediatr Cardiol 2017;38:941-5. 